\title{
Genome-wide SNPs reveal fine-scale population structure of Laodelphax striatellus in China using double-digest restriction site-associated DNA sequencing
}

\author{
Xingya Wang ${ }^{1}$, Bin $\mathrm{Lu}^{2}$, Lingyun Shao ${ }^{3}, \mathrm{Abid} \mathrm{Ali}^{4}$, Fengquan $\mathrm{Yu}^{3}$, Mei Yang ${ }^{3}$, Lei $\mathrm{Han}^{3}$, \\ and fuyu $\mathrm{Sun}^{3}$ \\ ${ }^{1}$ College of Plant Protection \\ ${ }^{2}$ Chengdu Institute of Biology, Chinese Academy of Science \\ ${ }^{3}$ Institute of Plant Protection \\ ${ }^{4}$ College of Life Sciences
}

June 6, 2020

\begin{abstract}
The small brown planthopper (SBPH), Laodelphax striatellus (Fallén) (Hemiptera: Delphacidae) is a most destructive rice pest which has caused serious economic losses in China. To effectively manage this pest, we investigated genomic polymorphism, evaluated genetic divergence and populations genetic structure of SBPH at 31 sampling sites in China using a population genomics approach. In this study, we generated over 2,813,221,369 high-quality paired-end reads (413,689.55 Mb) across 306 individuals derived from double-digest restriction site-associated DNA sequencing (ddRAD-seq), and 1925 reliable single nucleotides (SNPs) were detected with an average sequencing depth of $13.99 \times$. Overall, low levels of genetic diversity and strong genetic differentiation among all of populations were obtained (Global FST $=0.261$ ). Neighbour-joining dendrograms, Bayesian clustering methods, discriminant analysis of principal components (DAPC) and principal component analysis (PCA) revealed three genetically distinct groups: Southwestern group, Northern group and Eastern group. Furthermore, we also observed a high degree of admixture, widespread hybridization and gene flow among populations of Northern China. A Mantel test indicated a pattern of isolation-by-distance throughout China $(\mathrm{r}=0.211, \mathrm{P}=0.031)$. Neutrality test indicated SBPH has experienced a recent population expansion. Accordingly, our results provide new insights into the genetics of SBPH and thus contribute to develop effective management strategies for this pest.
\end{abstract}

\section{Hosted file}

Main manuscript.doc available at https://authorea.com/users/330525/articles/457278-genomewide-snps-reveal-fine-scale-population-structure-of-laodelphax-striatellus-in-chinausing-double-digest-restriction-site-associated-dna-sequencing 